\title{
Day-Ahead Scheduling of Wind Generation and Energy Storage
}

\author{
Mohamed Shaaban \\ Dept. of Electrical \& Electronic Engineering \\ Faculty of Engineering \\ Universiti Malaysia Sarawak \\ 94300 Kota Samarahan, Sarawak, Malaysia
}

\author{
Wen-Shan Tan and Md Pauzi Abdullah \\ Centre of Electrical Energy Systems \\ Institute of Future Energy \\ Universiti Teknologi Malaysia \\ 81310, Johor Bahru, Malaysia
}

\begin{abstract}
This paper presents a chance-constrained scheduling (CCS) approach for variable wind generation, in the day-ahead timescale, including energy storage. The day-ahead CCS utilizes the ramping of conventional generation as well as the dispatch of energy storage to enhance the load following and ramping support capabilities, to mitigate the impact of net load ramps. The proposed CCS approach is converted into an equivalent mixed-integer linear programming (MILP) expression with the aim to maintain the compatibility with commercially state-of-the-art optimization solvers. Numerical simulations, carried out on the IEEE RTS 96 test system with high penetration of wind power, indicate the effectiveness of the developed CCS formulation and highlight the competitive aspects of the proposed CCS approach.
\end{abstract}

Keywords- Generation scheduling, storage devices, wind generation, chance-constrained optimization, mixed-integer linear programming

\section{NOMENCLATURE}

\section{A. Functions}

$C_{\text {gen,it }}$ Total generation cost of unit $i$ at time $t$

$C_{\text {starnp,ut }}$ Total startup (shutdown) cost of unit $i$ at time $t$

$C_{C h r, j t} \quad$ Energy storage charging cost of unit $j$ at time $t$

$C_{\text {Dischr, }, ~}$ Energy storage discharging cost of unit $j$ at time $t$

\section{B. Parameters}

$G_{i}^{\max (\min )} \operatorname{Max}(\min )$ generation of unit $i[\mathrm{MW}]$

$D_{s t}^{m t} \quad$ Net load discrete realization level $s$ at time $\iota$ [MW]

$\varepsilon \quad$ Chance constraint confidence level

$\mathrm{Hr}_{i} \quad$ Incremental heat rate of unit $i$ [Btu/kWh]

$\pi_{i}^{g e n} \quad$ Incremental fuel cost of unit $i$ [\$/Btu]

$S U_{i l} \quad$ Start-up (shut-down) of step $l$ of unit $i[\$]$

$S R$ Spinning reserve at time $t$ [MW]

$q_{j}^{\text {max }, c(d)}$ Max charge (discharge) rate of storage $j$ [MWh]

$q_{1}^{\min , c(d)} \quad$ Min charge (discharge) rate of storage $j$ [MWh] $e_{j, m}^{\max / \min }$ Max (min) capacity of storage $j$ at time $m$ [MWh]

$E_{j}^{0} \quad$ Initial state of charge (SOC) of storage $j$ at the initial horizon [\%]

$\eta_{j}^{c(d)} \quad$ Efficiency rate to charge (discharge) of storage $j$

C. Binary variables at time $t$

$I_{u} \quad$ On/off status of units $i$

$z_{s t}, u_{s t} \quad$ Probabilistic auxiliary variable of level $s$

D. Continuous variables at time $t$

$r_{i j}^{u p(d n)}$ Ramp up (down) rate limit of unit $i$, in MWh

$G_{i i} \quad$ Dispatch of unit $i$, in MW

$q_{j t}^{c(d)} \quad$ Charge (discharge) rate of storage $j$ [MWh]

$e_{j i} \quad$ State of Charge (SOC) of storage $j[\%]$

$\bar{D}_{i}^{\text {net }} \quad$ Expected net load [MW]

flex $x_{i t}^{a n, \text { up }}$ Up flexibility of online unit $i$ at time $t$ [MW]

flex $x_{i}^{o f, u p}$ Up flexibility of offline unit $i$ at time $t$ [MW]

flex $_{i i}^{\text {on,an }}$ Down flexibility of online unit $i$ at time $t$ [MW]

flex $x_{i t}^{c h r}$ Up flexibility from charging of storage $j$ at time $t$ [MW]

flex dischr Down flexibility from discharging of storage $j$ at time $t$ [MW]

$p_{s t}^{\text {net }} \quad$ Probability of net load level $s$

\section{INTRODUCTION}

Over the last several decades, environmental issues and energy crises have accelerated the development of renewable energy across the world, especially wind power. This has introduced substantial uncertainties into the system operator's decision-making process, especially with the high penetration of wind power, causing power system operation paradigms, especially generation scheduling, to face new challenges [1]. A flexible grid system is needed to even out the variability and

This work was supported by the Universiti Malaysia Sarawak (UNIMAS), under Grant No. F02/SpGS/1544/2017. 